\title{
Fully developed forced convection of the Phan-Thien-Tanner fluid in ducts with a constant wall temperature
}

\author{
P.M. Coelho ${ }^{\mathrm{a}, *}$, F.T. Pinho ${ }^{\mathrm{a}}$, P.J. Oliveira ${ }^{\mathrm{b}}$ \\ ${ }^{a}$ Centro de Estudos de Fenómenos de Transporte, Departamento de Engenharia Mecânica, Faculdade de Engenharia, \\ Universidade do Porto, Rua Roberto Frias, 4200-465 Porto, Portugal

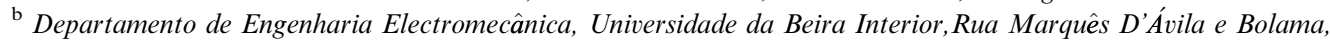 \\ 6200-001 Covilhã, Portugal
}

Received 19 January 2001; received in revised form 20 July 2001

\begin{abstract}
Two problems of laminar-forced convection in pipes and channels, under fully developed conditions, are solved for an imposed constant temperature at the wall, with fluids obeying the simplified Phan-Thien-Tanner (SPTT) model. The fluid properties are taken as constants and axial conduction is negligible. The first case represents the asymptotic behaviour of the Graetz problem for the SPTT fluid, i.e., equilibrium between axial convection and radial conduction of thermal energy with negligible viscous dissipation. The solution is given by an analytical expression but it is only approximate (within $0.3 \%$ ) as it was obtained with an algebraic method based on successive approximations. The second problem has an exact analytical solution representing the equilibrium between viscous dissipation and radial heat conduction, with negligible axial convection and a constant wall temperature. (c) 2002 Elsevier Science Ltd. All rights reserved.
\end{abstract}

\section{Introduction}

The simplified Phan-Thien-Tanner (SPTT) constitutive equation is a reduced version of the full PTT model of Phan-Thien and Tanner [1], which was derived from considerations of network theory and is given by Eq. (1). The simplification involved is to consider only affine motions of the polymer molecules relative to the continuum, with the consequence that the parameter $\xi$, that quantifies the slip velocity between the molecular network and the continuum medium, must be set to zero.

$Y(\operatorname{tr} \tau, T) \tau+\lambda\left(\stackrel{\nabla}{\tau}+\xi \mathbf{D} \cdot \tau+\xi \tau \cdot \mathbf{D}^{\mathrm{T}}\right)=2 \eta \mathbf{D}$.

In Eq. (1) $\stackrel{\nabla}{\tau}$ stands for Oldroyd's upper convected derivative of the stress tensor $\tau$, as defined by Eq. (2), $\lambda$ is a relaxation time, $\eta$ is a viscosity coefficient and $\mathbf{D}$ is the rate of strain tensor.

\footnotetext{
${ }^{*}$ Corresponding author.

E-mail addresses: pmc@fe.up.pt (P.M. Coelho), fpinho@ fe.up.pt (F.T. Pinho), pjpo@ubi.pt (P.J. Oliveira).
}

$\stackrel{\nabla}{\tau}=\frac{\mathrm{D} \tau}{\mathrm{D} t}-\tau \cdot \nabla \mathbf{u}-\nabla \mathbf{u}^{T} \cdot \tau$

The stress-coefficient function $Y$ is related to the rate of destruction of junctions in the molecular network and can be decoupled as

$Y(\operatorname{tr} \tau, T)=\phi(T) f(\operatorname{tr} \tau)$,

where $T$ is the temperature and $\operatorname{tr} \tau$ is the trace of the stress tensor $\tau$. Following Phan-Thien [2] the function $\phi(T)$ is arbitrarily set to unity at the reference temperature at which the material parameters of the model are determined, but here we generalise this assumption and so we do not consider thermal effects on the stress coefficient function $Y$.

The stress-dependent part of the stress-coefficient function has the exponential form:

$f(\operatorname{tr} \tau)=\exp \left(\frac{\varepsilon \lambda}{\eta} \operatorname{tr} \tau\right)$

which can be linearised as:

$f(\operatorname{tr} \tau)=1+\frac{\varepsilon \lambda}{\eta} \operatorname{tr} \tau$ 


\begin{tabular}{|c|c|c|c|}
\hline \multicolumn{2}{|c|}{ Nomenclature } & $\bar{T}_{\mathrm{I}}$ & bulk temperature at a reference location \\
\hline$a$ & $\begin{array}{l}\text { non-dimensional viscoelastic parameter, Eqs. } \\
\text { (12) and (42) }\end{array}$ & $\begin{array}{l}\mathbf{u} \\
U\end{array}$ & $\begin{array}{l}\text { velocity vector } \\
\text { cross-sectional average velocity }\end{array}$ \\
\hline $\begin{array}{l}\mathrm{Br} \\
\mathrm{c}\end{array}$ & $\begin{array}{l}\text { Brinkman number, Eq. (20) } \\
\text { specific heat }\end{array}$ & $U_{\mathrm{N}}$ & $\begin{array}{l}\text { cross-sectional average velocity for a New- } \\
\text { tonian fluid }\end{array}$ \\
\hline D & rate of strain tensor & $W e$ & Weissenberg number $(\lambda U / R)$ \\
\hline$D_{\mathrm{H}}$ & hydraulic diameter & $x$ & longitudinal coordinate \\
\hline$h$ & heat transfer coefficient & $Y$ & stress coefficient function \\
\hline$H$ & half-width of channel & $\alpha$ & thermal diffusivity \\
\hline$k$ & thermal conductivity & $\varepsilon$ & extensional parameter of PTT model \\
\hline $\mathrm{Nu}$ & Nusselt number & $\Phi$ & viscous dissipation function \\
\hline$p$ & pressure & $\eta$ & viscosity coefficient of PTT model \\
\hline$P e$ & Peclet number $\left(D_{\mathrm{H}} U / \alpha\right)$ & $\lambda$ & relaxation time of PTT model \\
\hline $\mathrm{Pr}$ & Prandtl number $(\eta c / k)$ & $\theta$ & non-dimensional temperature, Eq. (21) \\
\hline$\dot{q}_{\mathrm{w}}$ & heat flux at the wall & $\rho$ & density \\
\hline$r$ & radial coordinate & $\tau$ & stress tensor \\
\hline$R$ & pipe radius & $\xi$ & slip parameter of PTT model \\
\hline$T$ & temperature & * & refers to dimensionless quantities \\
\hline $\bar{T}$ & cross-sectional average temperature & & \\
\hline $\bar{T}_{\mathrm{i}}$ & perature at in & & \\
\hline
\end{tabular}

when the factor in brackets is small. This linearisation is adequate when the molecular deformation is small as in weak flows, following Tanner's [3] classification. According to this criteria, the pipe flow that is analysed here is a weak flow, i.e. it has a very limited amount of extensional molecular deformation and so Eq. (5) results in values in close agreement to those given by Eq. (4). The pipe flow is a shear-dominated flow for which most nonlinear models, such as the SPTT model, provide accurate descriptions of shear properties of polymer melts, as demonstrated by Peters et al. [4]. The exception here is the failure of SPTT to predict a non-zero second normal stress difference, which is proportional to $\xi$ in the full PTT model, but $N_{2}$ is totally irrelevant to the hydrodynamics of the fully developed pipe and channel flows and therefore is not considered.

In Eq. (5) $\varepsilon$ is a parameter related to the elongational behaviour of the model. It imposes an upper limit to the elongational viscosity that is proportional to the inverse of $\varepsilon$ [5, p. 227], and the upper-convected Maxwell model, which has an unbounded elongational viscosity in simple extensional flow, is recovered when $\varepsilon=0$.

The SPTT fluid is shear-thinning because of the term involving $\varepsilon$ in the stress-coefficient function $Y(\operatorname{tr} \tau)$, provided $\varepsilon$ is not too small. The degree of shear-thinning is not so accentuated as in the full PTT model, but the introduction of a nonzero $\xi$ is problematic because it causes a maximum shear stress in the rheogram. In fact, and according to Larson [6], by adopting the Gordon-Schowalter derivative (i.e. by having $\xi \neq 0$ ) the full PTT model inherits the problems of the Johnson-Segalman model, one of them being the maximum in the flow curve as shown by Alves et al. [7].

The analytical hydrodynamic solutions of the SPTT pipe and channel flows were derived by Oliveira and Pinho [8] who subsequently performed the analysis of the corresponding heat transfer problem for imposed constant wall heat flux [9]. In this latter work, due account has been taken of the effect of viscous dissipation, which is important for very viscous polymer melts, and as example of a very useful result for polymer processing was the determination of the axial variation of bulk temperature in the adiabatic regime [10] (obtained by setting $\dot{q}_{\mathrm{w}}=0$ or $B r=\infty$ in Eq. (28) of [9]). This was found to depend on the pressure gradient exactly as a Newtonian fluid, i.e., the adiabatic regime for a SPTT fluid is independent of viscoelasticity.

The present work extends the analysis of Pinho and Oliveira [9] to other relevant thermally fully developed asymptotic cases in duct (pipe or channel) with an imposed constant wall temperature: (1) the equilibrium between viscous dissipation and radial conduction, and (2) the equilibrium of axial convection with radial conduction in the absence of viscous dissipation. The solution of the first problem is exact and both temperature profiles and Nusselt number expressions are given. The solution for the Nusselt number in the second problem is analytical but it is only approximate as it was obtained with a successive approximation method. It represents the limiting Nusselt number at long duct distances for the Graetz problem of the SPTT fluid. This second solution turns out to be rather more complex than that for the imposed wall heat flux case. 
In polymer injection and extrusion the polymer melts flow at high temperature and are subject to various heat transfer processes. The quality of the final product depends on the ability to avoid hot spots and instabilities during the manufacturing process, and this requires careful control of heat transfer and temperature profiles [11], as well as of the hydrodynamics in order to avoid undesirable effects such as spurt [12].

Polymer melt flows in industry are characterised by very low Reynolds numbers (of the order of $10^{-3}-10^{-5}$ ) and high Prandtl numbers $\left(10^{6}-10^{8}\right)$, thus leading to a very fast hydrodynamic flow development but rather slow thermal development. This combination yields a Peclet number $(P e \equiv P r R e)$ of the order of 3000 and it is known that full thermal development for Newtonian fluids, under conditions of hydrodynamic fully developed flow, requires normalised lengths (length/transverse size) of $0.05 \mathrm{Pe}$ [13]. In extrusion heads the length of the parallel zone is well shorter than this critical length, but it is located at the end of a long, slightly tapered, duct where the flow is likely to be close to being thermal and hydrodynamic fully developed. In fact, in the downstream half of long tapered ducts the fully developed solution may also be a good approximation to the true prevailing conditions.

In the following section the problem is formulated, the hydrodynamic solution is presented and the analytical procedure required to solve the heat transfer problem, with constant wall temperature, is outlined. In Section 3, the solutions obtained under the two asymptotic assumptions are presented and discussed for the pipe flow case. Before closure, the corresponding solutions for the channel flow are given without details in Section 4.

\section{Formulation of the problem}

\subsection{Fluid model and assumptions}

Following the simplifications to the full PTT model described in the previous section, the fluid model under investigation is

$$
\left(1+\frac{\varepsilon \lambda}{\eta} \operatorname{tr} \tau\right) \tau+\lambda \tau=2 \eta \mathbf{D}
$$

with $\stackrel{\nabla}{\tau}$ given by Eq. (2).

The flow is assumed to be steady and laminar, under fully developed hydrodynamic and fully developed thermal conditions. The fluid properties are taken as constant and so no dependence of properties and model parameters on temperature will be considered. The boundary condition is that of an imposed constant temperature at the duct walls, $T_{\mathrm{w}}$. Two flow geometries are considered, namely the plane case (channel flow) and the axisymmetric case (pipe flow), but the details of the derivation are given only for the pipe flow. At the end of the paper the solution for the channel flow is presented without further comments.

Since polymer melts and concentrated polymer solutions are very viscous and the industrial flows frequently involve large velocity gradients, viscous dissipation effects will be considered in part of the analysis.

We further make the simplifying assumption of isotropic thermal and thermodynamic properties, although it is known that they are anisotropic due to their intimate relation to molecular structure. Consideration of property isotropy is fairly close to reality because, as the polymer melts, its chains tend towards random configurations after some characteristic time of the fluid. In this respect we follow previous heat transfer work relevant to polymer melts cited by Agassant et al. [10], Tadmor and Gogos [11] and Bird et al. [14], who showed that consideration of property isotropy did not seriously affected the results.

It is further assumed that Fourier's law of heat conduction is valid and that the internal energy and thermal conductivity do not depend explicitly on the velocity gradient or other kinematic quantities. For dilute polymer solutions there is some evidence that thermal conductivity may depend weakly on shear rate [15] (of the order of $10-20 \%$ over a range of $40 \leqslant \dot{\gamma} \leqslant 510 \mathrm{~s}^{-1}$ ), but the authors are unaware of similar work for concentrated polymer solutions and polymer melts. All these are standard, reasonable assumptions in heat transfer calculation of non-Newtonian fluids as discussed in Bird et al. [14, Section 4.4], Tanner [3, Section 9.1] and Agassant et al. [10, Chapter 2].

With the above assumptions the hydrodynamic and thermal problems become fully decoupled. The hydrodynamic solution is presented first, followed by the solution for the thermal problem, which is the focus of the present work.

\subsection{Hydrodynamic solution}

The hydrodynamic solution for the pipe flow was derived by Oliveira and Pinho [8] who arrived at the following velocity profile:

$$
\begin{aligned}
u^{*} \equiv & \frac{u}{U} \\
= & 2 \frac{U_{\mathrm{N}}}{U}\left[1-\left(\frac{r}{R}\right)^{2}\right]\left\{1+16 \varepsilon W e^{2}\left(\frac{U_{\mathrm{N}}}{U}\right)^{2}\right. \\
& \left.\times\left[1+\left(\frac{r}{R}\right)^{2}\right]\right\} .
\end{aligned}
$$

The non-dimensional group $W e=\lambda U / R$ is the Weissenberg number, a measure of the level of elasticity in the fluid and is based on the cross-sectional average 
velocity $U$ for the PTT fluid. $U_{\mathrm{N}}$ is the average velocity for a Newtonian fluid flowing under the same pressure gradient $\mathrm{d} p / \mathrm{d} x$

$U_{\mathrm{N}} \equiv \frac{-(\mathrm{d} p / \mathrm{d} x) R^{2}}{8 \eta}$

and was shown to be given by:

$\frac{U_{\mathrm{N}}}{U}=\frac{432^{1 / 6}\left(\delta^{2 / 3}-2^{2 / 3}\right)}{6 b^{1 / 2} \delta^{1 / 3}}$ with

$\delta=\left(3^{3} b+4\right)^{1 / 2}+3^{3 / 2} b^{1 / 2}$ and $b=\frac{64}{3} \varepsilon W e^{2}$.

The radial profiles of the shear stress $\tau_{x r}$ and the corresponding shear rate $\mathrm{d} u / \mathrm{d} r$ are also needed:

$\tau_{x r}^{*} \equiv \frac{\tau_{x r}}{4 \eta U / R}=-\frac{U_{\mathrm{N}}}{U}\left(\frac{r}{R}\right)$,

$\frac{\mathrm{d} u / \mathrm{d} r}{4 U / R}=-\frac{U_{\mathrm{N}}}{U}\left(\frac{r}{R}\right)\left[1+32 \varepsilon W e^{2}\left(\frac{U_{\mathrm{N}}}{U}\right)^{2}\left(\frac{r}{R}\right)^{2}\right]$.

It will be convenient for the foregoing analysis to define a modified non-dimensional group as

$a \equiv 16 \varepsilon W e^{2}\left(\frac{U_{\mathrm{N}}}{U}\right)^{2}$

which gives a measures of both the extensional and the elastic properties of the fluid. It should be recalled here from the hydrodynamic solution [8] that normal stresses will depend directly on $W e$ and inversely on some power of $\varepsilon W e^{2}$, whereas the shear stress will depend exclusively on $\varepsilon W e^{2}$. The dependence on $W e$ is solely a normal stress effect but the dependence on $\varepsilon W e^{2}$ combines a normal stress effect with the elongational parameter, and this combination imparts a shear-thinning behaviour to the viscosity function. It is in this context that the expression shear-thinning is used henceforth in the paper when referencing to the effect of $\varepsilon W e^{2}$.

\subsection{Heat transfer procedure}

The equation to be solved is the energy transport equation for the axisymmetric flow case with viscous dissipation, but without internal heat sources and negligible axial conduction:

$k \frac{1}{r} \frac{\partial}{\partial r}\left(r \frac{\partial T}{\partial r}\right)=\rho c u \frac{\partial T}{\partial x}-\Phi$,

where $k, \rho$ and $c$ stand for the thermal conductivity, density and specific heat of the fluid and $\Phi$ is the dissipation function. For the pipe flow this dissipation function involves only the shear stress and shear rate,

$\Phi \equiv \tau_{i j} \frac{\partial u_{i}}{\partial x_{j}}=\tau_{x r} \frac{\partial u}{\partial r}$.
The temperature $T$ depends on the radial $(r)$ and axial positions $(x)$ and $u$ stands for the axial velocity component. The relevant boundary conditions are symmetry at the axis:

$\left.\frac{\partial T}{\partial r}\right|_{r=0}=0$

and an imposed constant temperature at the wall

$T_{r=R}=T_{\mathrm{w}}$.

Next, we non-dimensionalise Eq. (13) by scaling lengths with the pipe radius $\left(r^{*} \equiv r / R, x^{*} \equiv x / R\right)$, velocity with the average velocity $\left(u^{*} \equiv u / U\right)$ and temperature as

$T^{*} \equiv \frac{T_{\mathrm{w}}-T}{T_{\mathrm{w}}-\bar{T}_{\mathrm{i}}}$,

where $\bar{T}_{\mathrm{i}}$ represents the bulk temperature at the inlet. The resulting dimensionless energy equation is

$\frac{1}{r^{*}} \frac{\partial}{\partial r^{*}}\left(r^{*} \frac{\partial T^{*}}{\partial r^{*}}\right)-4 B r \tau_{x r}^{*} \frac{\partial u^{*}}{\partial r^{*}}=\frac{P e}{2} u^{*} \frac{\partial T^{*}}{\partial x^{*}}$,

which expresses the so-called Graetz problem extended to account for viscous dissipation [16]. In Eq. (18) the Peclet number $P e$ is defined by

$P e \equiv \operatorname{PrRe}=\frac{\rho c U 2 R}{k}$

and the Brinkman number $\mathrm{Br}$ (following the original definition of Dryden, see [16]), by:

$B r \equiv \frac{\eta U^{2}}{k\left(T_{\mathrm{w}}-\bar{T}_{\mathrm{i}}\right)}$.

For Newtonian fluids the solution of Eq. (18) requires transformation of variables followed by such techniques as separation of variables or Lévêque analysis, and was obtained by Brinkman [18] and $\mathrm{Ou}$ and Cheng [19], amongst others (see also [14, Chapter 5] and [20]). For the SPTT fluid, the solution of Eq. (18) is rather more complex, because of the dependence on the Weissenberg number, and is not attempted here. We concentrate instead on obtaining two asymptotic solutions.

For such asymptotic solutions, it is convenient to normalise differently the axial convective term and for that purpose we introduce a non-dimensional temperature $\theta$ as

$\theta=\frac{T_{\mathrm{w}}-T}{T_{\mathrm{w}}-\bar{T}}$,

where $\bar{T}$ is the bulk temperature, so that the axial temperature gradient becomes:

$\frac{\partial T}{\partial x}=-\left(T_{\mathrm{w}}-\bar{T}\right) \frac{\partial \theta}{\partial x}+\theta \frac{\mathrm{d} \bar{T}}{\mathrm{~d} x}$. 
One possible advantage of using $\theta$ is that $\partial \theta / \partial x=0$ in fully developed thermal flow situations [13]. Hence, Eq. (22) reduces to

$\frac{\partial T}{\partial x}=\theta \frac{\mathrm{d} \bar{T}}{\mathrm{~d} x}$.

Introducing this result into the different non-dimensionalisation of the convective term of Eq. (13), and using again the starred quantities for the remaining normalisations, an alternative dimensionless energy equation is

$\frac{1}{r^{*}} \frac{\partial}{\partial r^{*}}\left(r^{*} \frac{\partial T^{*}}{\partial r^{*}}\right)=\frac{P e}{2} u^{*} \theta \frac{\mathrm{d} \bar{T}^{*}}{\mathrm{~d} x^{*}}+4 B r \tau_{x r}^{*} \frac{\partial u^{*}}{\partial r^{*}}$,

where all quantities on the right-hand side depend only on $r$, except the derivative of the bulk temperature which is independent of the radius, by definition.

The profile of the starred non-dimensional velocity of Eq. (7) is written as

$u^{*}=\frac{u}{U}=2 \frac{U_{\mathrm{N}}}{U}\left[1+a-r^{* 2}-a r^{* 4}\right]$

and the starred shear stress is defined in Eq. (10).

One of the important quantities to be obtained is the heat transfer coefficient under the form of a Nusselt number. This solution functional will also be useful to verify convergence of the iterative procedure to be explained below. The heat transfer coefficient is defined with dimensional quantities at the wall in the usual way:

$h \equiv \frac{\dot{q}_{\mathrm{w}}}{\bar{T}-T_{\mathrm{w}}}=\frac{-k(\partial T / \partial r)_{r=R}}{\bar{T}-T_{\mathrm{w}}}$

and the Nusselt number becomes

$N u \equiv \frac{2 R h}{k}=\frac{\left.2 R(\partial T / \partial r)\right|_{r=R}}{T_{\mathrm{w}}-\bar{T}}$

or, in dimensionless form

$N u=\frac{-\left.2\left(\partial T^{*} / \partial r^{*}\right)\right|_{r^{*}=1}}{\bar{T}^{*}}$.

We explain now the mixed analytical/numerical procedure utilised to solve Eq. (24) which requires application of a successive approximation method following Kays and Crawford [17, p. 96]. It starts, as a first approximation, with the $\theta$ profile for constant wall heat flux (given by Pinho and Oliveira [9, Eq. (32)]), which is substituted on the right-hand side of Eq. (24) together with the normalised profiles of velocity and shear stress. Since $\theta, u^{*}$ and $\tau_{x r}^{*}$ are polynomials in $r^{*}$, Eq. (24) is readily integrated for $T^{*}$ to obtain a corrected temperature profile for constant wall temperature. From this corrected $T^{*}$ profile, new bulk and $\theta$ temperatures are calculated using their definitions and a new expression for the Nusselt number is also evaluated. This newly corrected $\theta$ profile is then used to start the next iteration. The procedure is repeated in order to obtain systematically improved values of all quantities and of the Nusselt number which converges to a certain value. The procedure is stopped when the Nusselt number variation from successive approximations falls below a prescribed tolerance.

This method is applied to the solution of the developing thermal flow without viscous dissipation $(B r=0)$ with view to obtain an expression for the Nusselt number under fully developed conditions $(\partial \theta / \partial x=0)$. This constitutes the first asymptotic solution in this paper, henceforth termed the "fully developed thermal flow with negligible viscous dissipation".

A second asymptotic case is obtained when $\mathrm{d} \bar{T}^{*} / \mathrm{d} x^{*}$ vanishes in Eq. (24), whereby viscous dissipation is balanced by radial conduction only. The solution to this problem is easier and will also be given in the following section under the heading "equilibrium viscous dissipation flow".

\section{Solutions and discussion of results for pipe flow}

We give first the approximate solution for the problem of thermally fully developed flow without viscous dissipation (Eq. (24) with $B r=0$ ) and then we derive the exact solution for the problem of equilibrium between radial conduction and viscous dissipation (Eq. (24) with $\left.\mathrm{d} T^{*} / \mathrm{d} x^{*}=0\right)$.

\subsection{Fully developed thermal flow with negligible viscous dissipation}

As mentioned above, the initial guess for $\theta$ was taken as the solution to the problem with a constant wall heat flux obtained by Pinho and Oliveira [9] for negligible viscous dissipation $(B r=0)$

$$
\begin{aligned}
\theta\left(r^{*}\right)= & \left\{-\frac{1+a}{2} r^{* 2}+\frac{1}{8} r^{* 4}+\frac{a}{18} r^{* 6}+\frac{3}{8}+\frac{8}{18} a\right\} \\
& /\left[\frac{19}{54} a^{2}+\frac{17}{30} a+\frac{11}{48}\right] \frac{U_{\mathrm{N}}}{U} .
\end{aligned}
$$

The successive approximation method described in the previous section was applied until differences from consecutive iteration Nusselt numbers were less than $0.5 \%$. This happened at the end of the third iteration which differed from the second by less than $0.3 \%$ as can be assessed by inspection of Fig. 1. This figure plots $1-N u_{i+1} / N u_{i}$ as a function of $\varepsilon W e^{2}$, where the subscripts designate the iteration number and $N u_{q}$ is the initial Nusselt number corresponding to the condition of imposed wall heat flux, from [9]. It shows the quick convergence, with $N u_{2}$ already within $2 \%$ of $N u_{1}$, and that there is basically a decrease of one order of magnitude in the relative difference between consecutive Nusselt numbers. For the Newtonian fluid $(a=0)$, the Nusselt 


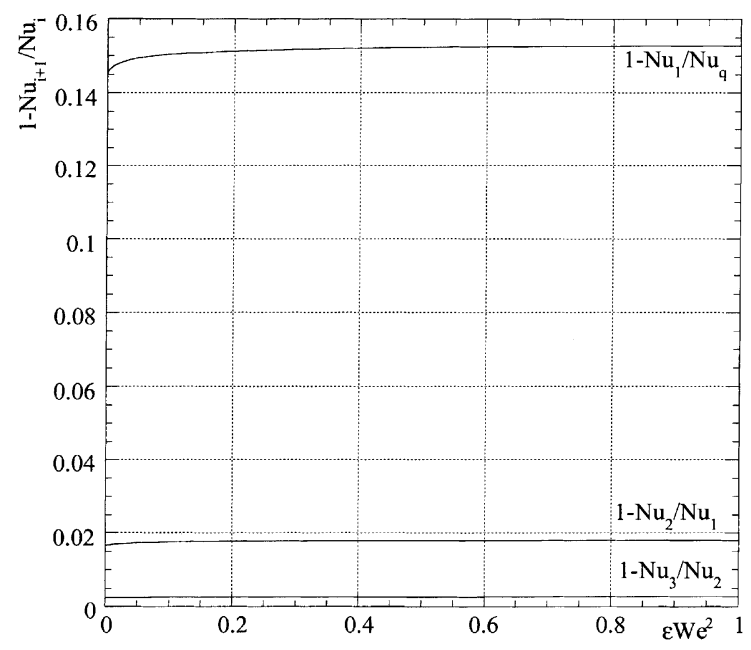

Fig. 1. Relative difference between Nusselt numbers at consecutive iterations as a function of $\varepsilon W e^{2}$.

number at the third iteration was well within $0.1 \%$ of the value quoted in the literature $(3.658$ in [17] or [16, 3.6568 quoted in p. 79]).

Tracking of the solution by algebraic means up to the third iteration (where an accuracy below $0.1 \%$ is estimated) was a tedious operation leading to lengthy equations in terms of powers of $r^{*}$. This will not be repeated here, and we shall content ourselves in giving the final "simplified" expressions for the temperature and
Nusselt number and analysing their variation from plots. The correctness of the approximate solution was checked by verifying that the same result is obtained when the method is started from different initial conditions, as reported in an internal report [21].

The normalised temperature profile is a long polynomial in $r^{*}$ and $a$ given by

$$
\begin{aligned}
T^{*}= & -\frac{P e}{2} \frac{\mathrm{d} \bar{T}^{*}}{\mathrm{~d} x^{*}}\left\{\left[0.9366 \sum_{i=0}^{4} \delta_{i} a^{i}+1.463 r^{* 2}\right.\right. \\
& \left.\left.\times \sum_{i=0}^{4}\left[\alpha_{i} a^{i} \times \sum_{j=0}^{11} \beta_{i j} r^{* 2 j}\right]\right] / \sum_{i=0}^{4} \gamma_{i} a^{i}\right\},
\end{aligned}
$$

where the coefficients $\alpha_{i}, \gamma_{i}$ and $\delta_{i}$ are presented in Table 1 and $\beta_{i j}$ in Table 2 .

The Nusselt number was found to be given by

$$
\begin{aligned}
N u= & 3.658\left\{\sum_{i=0}^{4} \gamma_{i} a^{i} /\left\{[ 1 + ( 4 / 3 ) a ] \left[2.4368 a^{5}\right.\right.\right. \\
& +10.172 a^{3}+17.004 a^{3}+14.230 a^{2} \\
& +5.9610 a+1]\}\},
\end{aligned}
$$

where the ratio of bulk velocities $U / U_{\mathrm{N}}$ has been be eliminated by means of the following expression from [8]

$$
\frac{U}{U_{\mathrm{N}}}=1+\frac{4}{3} a \text {. }
$$

\begin{tabular}{|c|c|c|c|c|c|}
\hline \multirow[t]{2}{*}{$j$} & \multicolumn{5}{|l|}{$i$} \\
\hline & 0 & 1 & 2 & 3 & 4 \\
\hline 0 & -1 & -1 & -1 & -1 & -1 \\
\hline 1 & $7.021 \times 10^{-1}$ & $6.302 \times 10^{-1}$ & $5.540 \times 10^{-1}$ & $4.730 \times 10^{-1}$ & $3.865 \times 10^{-1}$ \\
\hline 2 & $-3.376 \times 10^{-1}$ & $-2.418 \times 10^{-1}$ & $-1.456 \times 10^{-1}$ & $-4.898 \times 10^{-2}$ & $4.817 \times 10^{-2}$ \\
\hline 3 & $1.111 \times 10^{-1}$ & $4.659 \times 10^{-2}$ & $-1.096 \times 10^{-2}$ & $-6.102 \times 10^{-2}$ & $-1.029 \times 10^{-1}$ \\
\hline 4 & $-2.788 \times 10^{-2}$ & $-4.600 \times 10^{-4}$ & $1.819 \times 10^{-2}$ & $2.802 \times 10^{-2}$ & $2.895 \times 10^{-2}$ \\
\hline 5 & $4.865 \times 10^{-3}$ & $-3.383 \times 10^{-3}$ & $-6.130 \times 10^{-3}$ & $-4.104 \times 10^{-3}$ & $1.900 \times 10^{-3}$ \\
\hline 6 & $-5.602 \times 10^{-4}$ & $1.108 \times 10^{-3}$ & $6.501 \times 10^{-4}$ & $-1.109 \times 10^{-3}$ & $-3.354 \times 10^{-3}$ \\
\hline 7 & $2.814 \times 10^{-5}$ & $-1.861 \times 10^{-4}$ & $1.219 \times 10^{-4}$ & $4.953 \times 10^{-4}$ & $5.679 \times 10^{-4}$ \\
\hline 8 & 0 & $1.186 \times 10^{-5}$ & $-5.305 \times 10^{-5}$ & $-4.994 \times 10^{-5}$ & $8.634 \times 10^{-5}$ \\
\hline 9 & 0 & 0 & $4.802 \times 10^{-6}$ & $-1.091 \times 10^{-5}$ & $-3.870 \times 10^{-5}$ \\
\hline 10 & 0 & 0 & 0 & $1.854 \times 10^{-6}$ & 0 \\
\hline 11 & 0 & 0 & 0 & 0 & $6.745 \times 10^{-7}$ \\
\hline
\end{tabular}

Table 1

Coefficients $\alpha_{i}, \gamma_{i}$ and $\delta_{i}$ for pipe flow

\begin{tabular}{llllll}
\hline & 0 & 1 & 2 & 3 & 4 \\
\hline$\alpha_{i}$ & 0.614 & 2.779 & 4.711 & 3.546 & 1 \\
$\gamma_{i}$ & 1 & 4.7967 & 8.6410 & 6.9291 & 2.0869 \\
$\delta_{i}$ & 0.5258 & 2.4656 & 4.3405 & 3.4 & 1 \\
\hline
\end{tabular}

Table 2

Coefficient $\beta_{i j}$ for pipe flow 

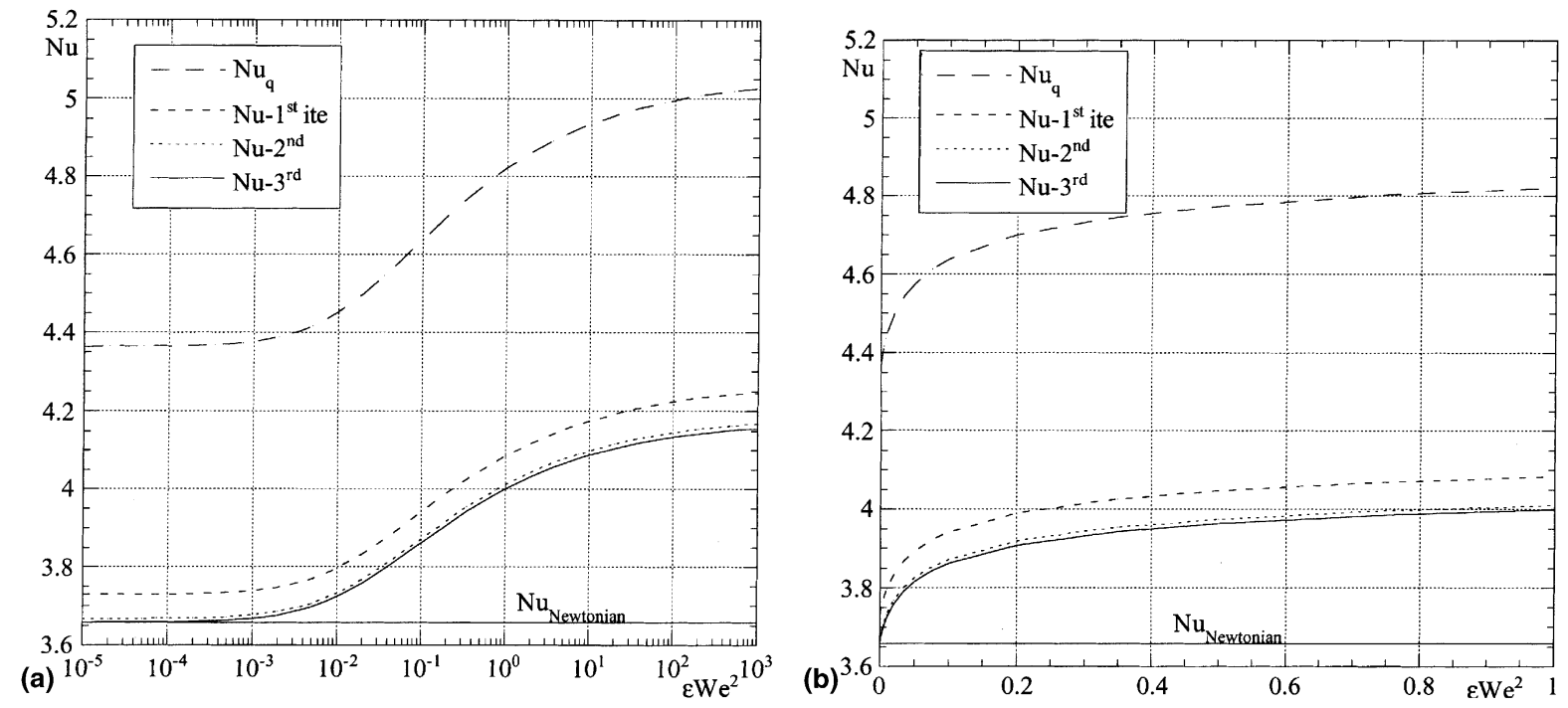

Fig. 2. Variation of the Nusselt number with $\varepsilon W e^{2}$. Dashed lines show values at consecutive iterations, starting from the case of imposed heat flux $\left(N u_{q}\right)$ : (a) in semi-log coordinates; (b) in linear coordinates.

The normalised temperature $\theta$, as defined by Eq. (21), is independent of axial location for thermally fully developed flow, as implied by the definition of this asymptotic condition. It can be obtained from a balance of energy at a cross-section, which shows that

$$
\left.\frac{\partial T^{*}}{\partial r^{*}}\right|_{r^{*}=1}=\frac{P e}{4} \frac{\mathrm{d} \bar{T}^{*}}{\mathrm{~d} x^{*}}
$$

hence from Eq. (28) we conclude that

$\bar{T}^{*}=-\frac{P e}{2} \frac{\mathrm{d} \bar{T}^{*}}{\mathrm{~d} x^{*}} \frac{1}{N u}$

and $\theta=T^{*} / \bar{T}^{*}$, with the numerator and denominator given by Eqs. (30) and (34), respectively.

The full lines in Figs. 2(a) and (b) show the resulting variation of Nusselt number with $\varepsilon W e^{2}$, which is the relevant viscoelastic number in these flows, in both linear and semi-log scales. The dashed lines in the figures help to show the iterative progression of Nusselt number and complement the information of Fig. 1. They are useful as a check on the correctness of the implementation of the numerical method, and clearly show that more iterations would bring a negligible improvement on the solution.

The Nusselt number varies between two asymptotes: at the limit of vanishing $\varepsilon W e^{2}$ it gives the well-known Newtonian value of 3.658 and, as $\varepsilon W e^{2}$ goes to infinity, it tends to 4.178 . There is thus a maximum increase of $14 \%$ in relation to the Newtonian solution, represented in the figure as a horizontal line. It is very unlikely that flows attain such high values of $\varepsilon W e^{2}$ and Fig. 2(b) presents the same information in linear coordinates and in a limited range, $\varepsilon W e^{2} \in[0,1]$. At $\varepsilon W e^{2}=1$ the Nus- selt number is already equal to four, representing an increase of $9.4 \%$ from the Newtonian value. As with the Nusselt number for constant wall heal flux, represented in the figures in long dashes, the Nusselt number increases with $\varepsilon W e^{2}$ due to the more pronounced shearthinning effect.

The variation of $\theta$ with radius is given in Fig. 3 for various values of the parameter $\varepsilon W e^{2}$. The variation with $\varepsilon W e^{2}$ is mild since both its numerator and denominator depend similarly on $\varepsilon W e^{2}$, a conclusion similar to that reached in the previous work for the case of imposed wall heat flux [9].

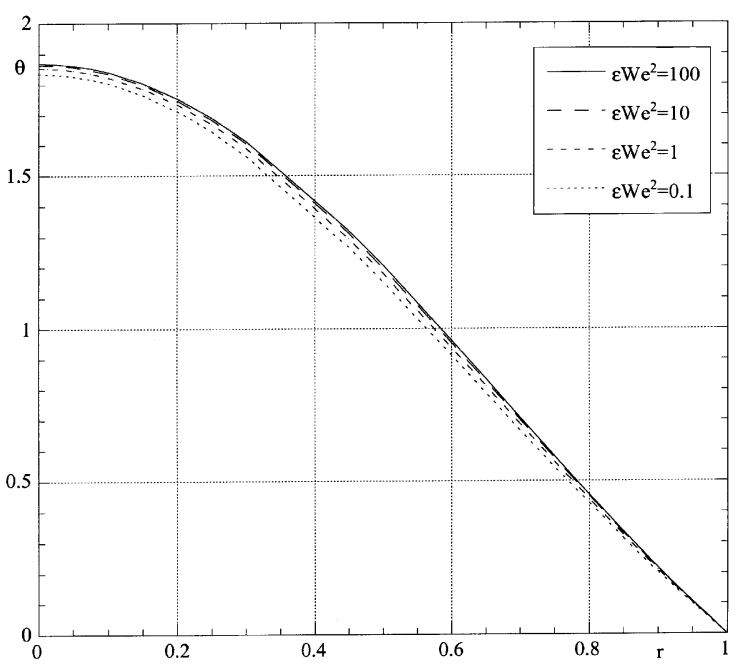

Fig. 3. Radial profiles of $\theta$ as a function of fluid elasticity. 
The practical use of the expressions for temperature (Eqs. (30) and (34)) is rather limited unless the variation of the bulk temperature with the axial coordinate is known. The successive approximation method used here cannot provide such gradient because it only gives the asymptotic radial variation. However, in the fully developed flow region, where $N u$ has reached its constant asymptotic value, it is possible to integrate the energy balance (Eq. (34)) yielding the following axial variation of bulk temperature

$\bar{T}^{*}=\bar{T}_{\mathrm{I}}^{*} \exp \left[-\frac{2 N u}{P e} x^{*}\right]$

from which its axial gradient is obtained

$\frac{\mathrm{d} \bar{T}^{*}}{\mathrm{~d} x^{*}}=-\frac{2 N u}{P e} \bar{T}_{\mathrm{I}}^{*} \times \exp \left[-\frac{2 N u}{P e} x^{*}\right]$.

If $x^{*}$ is the axial position relative to a reference location within the fully developed region, and $\bar{T}_{\mathrm{I}}$ is the bulk temperature at that reference location, then Eqs. (35) and (36) give exact values for the bulk temperature and its gradient; otherwise, if there is no certainty that $x^{*}$ lies in the fully developed region, then $\bar{T}_{\mathrm{I}}$ has to be estimated and relations (35) and (36) are only approximate.

\subsection{Equilibrium viscous dissipation flow}

We turn now to the second problem involving a balance between radial conduction and viscous dissipation under fully developed conditions. Substitution of the velocity profile (Eq. (25)) and the shear stress and shear rate profiles (Eqs. (10) and (11)) into Eq. (24) with $\mathrm{d} \bar{T}^{*} / \mathrm{d} x^{*}=0$, produces

$\frac{1}{r^{*}} \frac{\partial}{\partial r^{*}}\left(r^{*} \frac{\partial T^{*}}{\partial r^{*}}\right)=16 B r\left(\frac{U_{\mathrm{N}}}{U}\right)^{2}\left[2 a r^{* 4}+r^{* 2}\right]$,

which is of straightforward integration. The first integration gives the temperature gradient at the wall needed to obtain the Nusselt number

$$
\begin{aligned}
\left.\frac{\mathrm{d} T^{*}}{\mathrm{~d} r^{*}}\right|_{r^{*}=1} & =4 B r\left(\frac{U_{\mathrm{N}}}{U}\right)^{2} \frac{4 a+3}{3}=4 B r \frac{3}{4 a+3} \\
& =4 B r \frac{U_{\mathrm{N}}}{U},
\end{aligned}
$$

where use was made of expression (32). A second integration and imposition of the wall temperature boundary condition gives the temperature distribution

$T^{*}=B r\left(\frac{U_{\mathrm{N}}}{U}\right)^{2} \frac{\left[8 a\left(r^{* 6}-1\right)+9\left(r^{* 4}-1\right)\right]}{9}$

and the dimensionless bulk temperature becomes

$\bar{T}^{*}=-B r\left(\frac{U_{\mathrm{N}}}{U}\right)^{3} \frac{280 a^{2}+504 a+225}{270}$.
Finally, the Nusselt number is

$N u=\frac{240(4 a+3)^{2}}{280 a^{2}+504 a+225}$,

which gives the correct Newtonian value $N u=48 / 5$ for $a=0[16, \mathrm{p} .80]$. The important conclusion here is that the Nusselt number does not depend on the Brinkman number. The definition of $N u$ in Eq. (28), involves the ratio of two quantities that in this limiting problem depend linearly on the Brinkman number and consequently $\mathrm{Nu}$ becomes independent of $\mathrm{Br}$.

We know from the equivalent constant wall heat-flux problem [9] that viscous dissipation has a strong impact upon the heat transfer characteristics, and this is confirmed in the present case. For a Newtonian fluid the Nusselt number more than doubles, from 3.658 to 9.6, but this increase is accentuated for the viscoelastic fluid, as shown in the comparative plot of Fig. 4. For $\varepsilon W e^{2}=1$ the normalised heat transfer coefficient with viscous dissipation is already three times higher than that for negligible dissipation, and this ratio increases to the limiting value of 3.28 as $\varepsilon W e^{2} \rightarrow \infty(N u \rightarrow 13.714$ with dissipation, compared with $\mathrm{Nu} \rightarrow 4.178$ without dissipation).

Radial temperature profiles for these situations are plotted in Fig. 5. An increase in Brinkman number raises the temperature level linearly in the pipe section. Although the influence of viscous dissipation increases with flow elasticity, as seen in the Nusselt number plot of Fig. 4, the difference between the local and the wall temperatures (recall that $-T^{*} \propto T-T_{\mathrm{w}}$ ) decreases with $\varepsilon W e^{2}$, because the flow becomes increasingly shear-thinning due to normal stress effects and consequently viscous dissipation becomes progressively more localised in

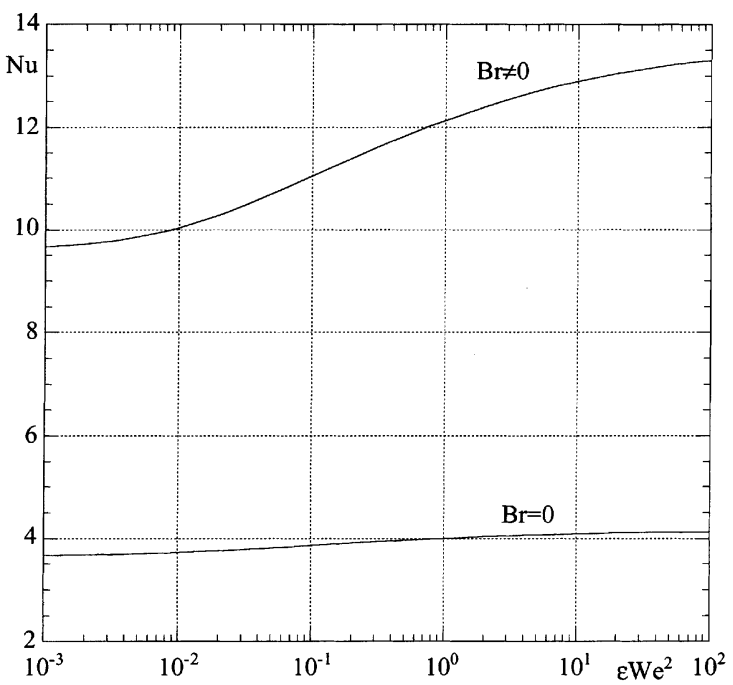

Fig. 4. Comparison between the Nusselt numbers for negligible viscous dissipation and the limiting equilibrium viscous dissipation condition, as a function of fluid elasticity $\left(\varepsilon W e^{2}\right)$. 


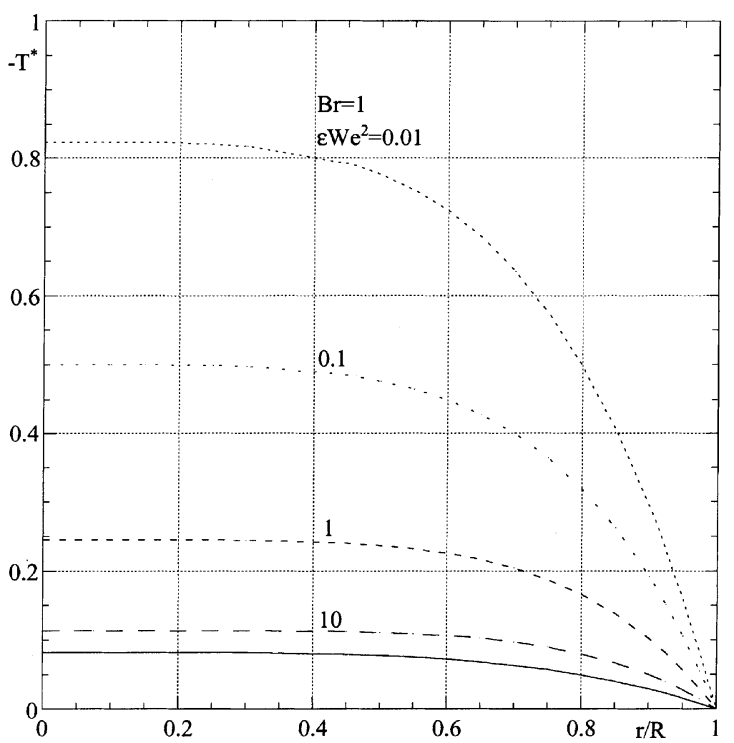

Fig. 5. Effect of viscoelasticity and Brinkman number on the radial temperature profiles in the equilibrium viscous dissipation problem. The full line is for $B r=0.1$ and $\varepsilon W e^{2}=0.01$.

the wall region and less pronounced in the core of the flow. Thus, since the heat is generated closer to the wall, it is evacuated easier without the need to heat the bulk of the flow. Note that the decrease in $-T^{*}$ with viscoelasticity induces a wider core of uniform temperature.

\section{Results for channel flow}

The solution of the two heat transfer problems for the channel flow case follows qualitatively that of the pipe flow, with some differences arising from the different forms of the energy equation and the velocity profile. The channel half-width is denoted $H$ and the parameter $a$ is now defined as:

$a \equiv 9 \varepsilon W e^{2}\left(\frac{U_{\mathrm{N}}}{U}\right)^{2}$ with $\frac{U}{U_{\mathrm{N}}}=1+\frac{6}{5} a$

and $W e=\frac{\lambda U}{H}$

following [8]. Results for the two cases previously addressed in the pipe geometry are presented next.
4.1. Fully developed thermal flow with negligible viscous dissipation

For the problem with negligible viscous dissipation, with both walls having the same temperature, the transverse distribution of temperature is given by Eq. (43):

$$
\begin{aligned}
T^{*}= & -\frac{P e}{2} \frac{\mathrm{d} \bar{T}^{*}}{\mathrm{~d} x^{*}}\left\{\left[0.6694 \sum_{i=0}^{4} \delta_{i} a^{i}+0.8604 y^{* 2}\right.\right. \\
& \left.\left.\times \sum_{i=0}^{4}\left[\alpha_{i} a^{i} \times \sum_{j=0}^{11} \beta_{i j} y^{* 2 j}\right]\right] / \sum_{i=0}^{4} \gamma_{i} a^{i}\right\} .
\end{aligned}
$$

After application of the successive approximation method, the Nusselt number is now found to be given by:

$$
\begin{aligned}
N u= & 6.8567\left\{\sum_{i=0}^{4} \gamma_{i} a_{i} /\left[( U / U _ { \mathrm { N } } ) \left[a^{5}+4.4810 a^{4}\right.\right.\right. \\
& \left.\left.\left.+8.0360 a^{3}+7.2093 a^{2}+3.2355 a+0.58111\right]\right]\right\}
\end{aligned}
$$

and the difference between the bulk and wall temperatures is

$\bar{T}^{*}=-\frac{P e}{2} \frac{\mathrm{d} \bar{T}^{*}}{\mathrm{~d} x^{*}} \frac{4}{N u}$.

The parameters $\alpha_{i}, \gamma_{i}$ and $\delta_{i}$ in Eqs. (43) and (44) are listed in Table 3 and $\beta_{i j}$ in Table 4.

For a Newtonian fluid $(a=0)$ the Nusselt number expression simplifies to 7.541 , the correct value from the literature $[16$, p. 155]).

\subsection{Equilibrium viscous dissipation flow}

For the other fully developed problem, that of the equilibrium between transverse conduction and heat production by viscous dissipation, the analytical solution of the energy equation produces the temperature profile of Eq. (46)

$T^{*}=3 B r\left(\frac{U_{\mathrm{N}}}{U}\right)^{2} \frac{\left[4 a\left(y^{*^{6}}-1\right)+5\left(y^{*^{4}}-1\right)\right]}{20}$.

Table 3

Coefficients $\alpha_{i}, \gamma_{i}$ and $\delta_{i}$ for channel flow

\begin{tabular}{llllll}
\hline & 0 & 1 & 2 & 3 & 4 \\
\hline$\alpha_{i}$ & 0.7348 & 3.1757 & 5.1461 & 3.7051 & 1 \\
$\gamma_{i}$ & 0.6391 & 2.8563 & 4.7902 & 3.5728 & 1 \\
$\delta_{i}$ & 0.6680 & 2.9532 & 4.8985 & 3.6132 & 1 \\
\hline
\end{tabular}


Table 4

Coefficients $\beta_{i j}$ for channel flow

\begin{tabular}{|c|c|c|c|c|c|}
\hline \multirow[t]{2}{*}{$j$} & \multicolumn{5}{|l|}{$i$} \\
\hline & 0 & 1 & 2 & 3 & 4 \\
\hline 0 & -1 & -1 & -1 & -1 & -1 \\
\hline 1 & $4.019 \times 10^{-1}$ & $3.580 \times 10^{-1}$ & $3.121 \times 10^{-1}$ & $2.641 \times 10^{-1}$ & $2.138 \times 10^{-1}$ \\
\hline 2 & $-1.316 \times 10^{-1}$ & $-8.722 \times 10^{-2}$ & $-4.240 \times 10^{-2}$ & $2.902 \times 10^{-3}$ & $4.873 \times 10^{-2}$ \\
\hline 3 & $2.646 \times 10^{-2}$ & $5.108 \times 10^{-3}$ & $-1.454 \times 10^{-2}$ & $-3.235 \times 10^{-2}$ & $-4.818 \times 10^{-2}$ \\
\hline 4 & $-4.688 \times 10^{-3}$ & $1.248 \times 10^{-3}$ & $5.271 \times 10^{-3}$ & $7.325 \times 10^{-3}$ & $7.346 \times 10^{-3}$ \\
\hline 5 & $5.547 \times 10^{-4}$ & $-7.411 \times 10^{-4}$ & $-1.071 \times 10^{-3}$ & $-5.030 \times 10^{-4}$ & $8.851 \times 10^{-4}$ \\
\hline 6 & $-5.047 \times 10^{-5}$ & $1.343 \times 10^{-4}$ & $2.968 \times 10^{-5}$ & $-2.769 \times 10^{-4}$ & $-6.938 \times 10^{-4}$ \\
\hline 7 & $1.769 \times 10^{-6}$ & $-1.733 \times 10^{-5}$ & $1.772 \times 10^{-5}$ & $6.124 \times 10^{-5}$ & $7.178 \times 10^{-5}$ \\
\hline 8 & 0 & $7.567 \times 10^{-7}$ & $-5.087 \times 10^{-6}$ & $-4.497 \times 10^{-6}$ & $1.066 \times 10^{-5}$ \\
\hline 9 & 0 & 0 & $3.078 \times 10^{-7}$ & $-1.072 \times 10^{-6}$ & $-3.972 \times 10^{-6}$ \\
\hline 10 & 0 & 0 & 0 & $1.177 \times 10^{-7}$ & 0 \\
\hline 11 & 0 & 0 & 0 & 0 & $4.137 \times 10^{-8}$ \\
\hline
\end{tabular}

The difference between the bulk and wall temperatures is given by

$\bar{T}^{*}=-24 B r\left(\frac{U_{\mathrm{N}}}{U}\right)^{3} \frac{54 a^{2}+110 a+55}{1925}$

and the Nusselt number is quantified by

$N u=\frac{1925(1+(6 / 5) a)^{2}}{2\left(54 a^{2}+110 a+55\right)}$.

The variation of the Nusselt number with $\varepsilon W e^{2}$ and the temperature profiles across the channel have been checked to follow the same trends as those for the pipe flow cases discussed in Section 3.

\section{Conclusions}

The steady, laminar flow of the simplified PhanThien-Thanner model fluid, in pipes and channels, was investigated for the condition of an imposed constant wall temperature and under fully developed thermal and hydrodynamic conditions. The two possible cases were investigated and results were presented for the radial/ traverse profile of normalised temperature and for the Nusselt number as a function of the relevant non-dimensional Brinkman number and the product of $\varepsilon$ with the square of Weissenberg number.

The first fully developed solution pertained to the equilibrium between axial convection and radial conduction of thermal energy, with negligible viscous dissipation, and here it was observed that an increase in fluid elasticity (as measured by $\sqrt{\varepsilon} W e$, to be more precise) raised the normalised heat transfer coefficient by at most $14 \%$ due to the increased level of shear-thinning behaviour.

For the second thermally fully developed problem, where there is equilibrium between radial conduction of energy and heat production by viscous effects, viscous dissipation is responsible for the increase in the Nusselt number which is more pronounced the more elastic the fluid is. Again, that was found to be associated with the more intense shear-thinning fluid behaviour: shearthinning is enhanced by increasing $\sqrt{\varepsilon} W e$, which was seen to raise shear close to the wall while simultaneously decreasing them in the core of the flow. Since viscous dissipation is proportional to the shear rate, as shearthinning is enhanced the internal production of heat increases nearer to the wall where heat is evacuated, and decreases in the core of the duct, thus the thermal resistance decreases and the Nusselt number raises.

\section{References}

[1] N. Phan-Thien, R.I. Tanner, A new constitutive equation derived from network theory, J. Non-Newtonian Fluid Mech. 2 (1977) 353-365.

[2] N. Phan-Thien, A nonlinear network viscoelastic model, J. Rheol. 22 (1978) 259-283.

[3] R.I. Tanner, Engineering Rheology, Clarendon Press, Oxford, 1985.

[4] G.W.M. Peters, J.F.M. Schoonen, F.P.T. Baaijens, H.E.H. Meijer, On the performance of enhanced constitutive models for polymer melts in a cross-slot flow, J. NonNewtonian Fluid Mech. 82 (1999) 387-427.

[5] R.R. Huilgol, N. Phan-Thien, Fluid Mechanics of Viscoelasticity, in: Rheology Series, vol. 6, Elsevier, Amsterdam, 1997.

[6] R.G. Larson, Constitutive Equations for Polymer Melts and Solutions, Boston, Butterworths, 1988.

[7] M.A. Alves, F.T. Pinho, P.J. Oliveira, Theoretical study of steady duct flows of the full Phan-Thien-Tanner fluid, J. Non-Newtonian Fluid Mech. 101 (1-3) (2001) 55-76.

[8] P.J. Oliveira, F.T. Pinho, Analytical solution for fully developed channel and pipe flow of Phan-Thien-Tanner fluids, J. Fluid Mech. 387 (1999) 271-280.

[9] F.T. Pinho, P.J. Oliveira, Analysis of forced convection in pipes and channels with the simplified Phan-Thien-Tanner fluid, Int. J. Heat Mass Transfer 43 (2000) 2273-2287. 
[10] J.F. Agassant, P. Avenas, J.P.H. Sergent, P.J. Carreau, Polymer Processing Principles and Modelling, Hansen Publishers, Munich, Germany, 1991.

[11] Z. Tadmor, C.G. Gogos, Principles of Polymer Processing, first ed., Wiley, New York, 1979.

[12] R.W. Kolkka, D.S. Malkus, M.G. Hansen, G.R. Ierley, R.A. Worthing, Spurt phenomena of the Johnson-Segalman fluid and related models, J. Non-Newtonian Fluid Mech. 29 (1988) 303-335.

[13] E.R.G. Eckert, R.M. Drake Jr., Analysis of Heat and Mass Transfer, first ed., McGraw-Hill, New York, 1972.

[14] R.B. Bird, R.C. Armstrong, O. Hassager, Fluid Dynamics. Vol. 1: Dynamics of Polymeric Liquids, second ed., Wiley, New York, 1987.

[15] M. Kostic, H. Tong, Investigation of thermal conductivity of a polymer solution as a function of shear rate, Paper \# 199 presented at 1999 IMECE, Fluids Engineering Division, Nashville, TN, USA, ASME Heat Transfer Section.

[16] R.K. Shah, A.L. London, Laminar Flow Forced Convection in Ducts, Academic Press, New York, 1978.
[17] W.M. Kays, M.E. Crawford, Convective Heat and Mass Transfer, second ed., McGraw-Hill, New York, 1980.

[18] H.C. Brinkman, Heat effects in capillary flow I, Appl. Sci. Res. A 2 (1951) 120-124.

[19] J.W. Ou, K.C. Cheng, Viscous dissipation effects on thermal entrance heat transfer in laminar and turbulent pipe flows with uniform wall temperature, Paper ASME 74-HT-50. AIAA/ASME 1974 Thermophysics and Heat Transfer Conference, Boston, MA, 1974, July $15-17$.

[20] G.M. Brown, Heat and mass transfer in a fluid in laminar flow in a circular or flat conduit, AIChE J. 6 (1960) 179-183.

[21] P.M. Coelho, F.T. Pinho, P.J. Oliveira, 2001. Constant wall temperature forced convection in ducts with a simplified Phan-Thien-Tanner fluid. Internal report of Centro de Estudos de Fenómenos de Transporte, Faculdade de Engenharia da Universidade do Porto, Portugal and Departmento de Engenharia Electromecânica, Universidade da Beira Interior, Covilhã, Portugal. 